\title{
Pontos Chaves para Adoção de Uma Arquitetura Orientada a Serviços: Uma Análise Comparativa de Modelos de Maturidade SOA da Indústria
}

\author{
José Jorge Lima Dias Jr. ${ }^{1,2}$, Joyce Aline P. de Oliveira ${ }^{1}$, Silvio R. de Lemos Meira ${ }^{2}$ \\ ${ }^{1}$ Centro de Ciências Aplicadas e Educação - Universidade Federal da Paraíba (UFPB) \\ Rio Tinto - PB - Brasil \\ ${ }^{2}$ Centro de Informática - Universidade Federal de Pernambuco (UFPE) \\ Recife - PE - Brasil \\ \{jorge, joyce.aline\}@dce.ufpb.br, srlm@cin.ufpe.br
}

\begin{abstract}
Adoption of Service-Oriented Architecture (SOA) has become a common practice in companies that aims at solving problems of flexibility to change and application integration. Although SOA promises to solve such problems, its adoption is not trivial in practice since it requires a process of adaptation and an organizational and technological restructuring. In this context, several SOA Maturity Models have been proposed aiming to aid the adoption process. Hence, this paper presents a comparative analysis among SOA Maturity Models proposed by industry, presenting similarities and differences among them in order to identify key points that can aid in SOA adoption.
\end{abstract}

\begin{abstract}
Resumo. A adoção de SOA tem se tornado prática frequente em organizações que buscam resolver problemas de flexibilidade a mudanças e de integração entre suas aplicações. Embora SOA, teoricamente, resolva tais problemas, sua adoção na prática não consiste em uma atividade trivial, pois requer um processo de adaptação e de reestruturação organizacional e tecnológica. Neste contexto, vários modelos de maturidade SOA vem sendo propostos com o objetivo de auxiliar este processo de adoção. Diante disto, este artigo apresenta uma análise comparativa entre modelos de maturidade SOA propostos na indústria, apresentando suas semelhanças e diferenças a fim de identificar pontos chaves que auxiliem na adoção de SOA.
\end{abstract}

\section{Introdução}

Torna-se cada vez mais difícil manter-se indiferente à expansão decorrente dos avanços da globalização e da tecnologia. A dinâmica do mercado tem exigido uma maior integração entre organizações independentemente de políticas, culturas ou espaço geográfico. Empresas fornecedoras de produtos distintos mantêm parcerias com diferentes tipos de organizações compartilhando diversos tipos de informações e serviços. Muitas dessas organizações possuem uma miscelânea de sistemas, aplicações e arquiteturas com diferentes idades, tecnologias e plataformas [Josuttis 2007]. Além disso, novos softwares são adquiridos de acordo com a necessidade da empresa, 
aumentando ainda mais a complexidade do seu sistema, tornando-o cada vez mais diversificado e desintegrado.

Paralelamente à adição de novas tecnologias, ocorre a constante alteração dos processos de negócio das empresas, seja pela aquisição de novos serviços, seja pela necessidade de melhoria das operações internas ou ainda pela busca de alinhamento entre o negócio e a tecnologia da informação. $O$ fato é que o mercado tem forçado as empresas a reagir rapidamente às mudanças, exigindo a adaptação dos seus processos de forma contínua [Niemann et al. 2008].

A Arquitetura Orientada a Serviços (SOA - Service-Oriented Architecture) surge como uma resposta a este desafio apresentando-se como um paradigma para a organização e utilização de funcionalidades distribuídas que podem estar sob o domínio de proprietários distintos [OASIS 2006].

Embora a adoção de SOA, resolva, teoricamente, problemas de flexibilidade às mudanças e de integração nas organizações, sua implantação na prática envolve riscos e requer um alto custo, além de um longo processo de adaptação e de reestruturação tecnológica e organizacional, de modo que não deve ser realizada sem um planejamento prévio e sem um objetivo definido [Nasr et al. 2010].

As abordagens existentes para o planejamento sistemático de adoção, implantação e continuidade de uma Arquitetura Orientada a Serviços são denominadas de Modelos de Maturidade em SOA e possibilitam à empresa analisar o nível de alinhamento com os conceitos relacionados a SOA, tornando possível avaliar o quanto é necessário evoluir para a obtenção na íntegra desta abordagem.

A fim de identificar quais são os pontos chaves para ajudar na adoção de SOA, foi realizada uma análise comparativa entre os principais modelos de maturidade SOA propostos na indústria. Os modelos analisados foram: IBM, ORACLE, HP, SONIC, BEA-IT e OPEN GROUP. A escolha destes modelos é justificada pelo fato de serem propostos por grandes organizações que adquiriram experiência relevante ao longo dos anos concernente a implantação de SOA. Além disso, estas organizações oferecem ferramentas apropriadas que apóiam a adoção desta abordagem.

A identificação dos pontos chaves para a adoção de SOA justifica-se por ser observado que, em alguns casos, muitas empresas não conhecem inicialmente o que deve ser levado em consideração. Neste contexto, este artigo tenciona descrever tais pontos com o intuito de auxiliar as organizações a entender o que deve ser abordado para que haja sucesso no alcance de uma Arquitetura Orientada a Serviços.

Este artigo está organizado da seguinte forma: a seção 2 apresenta resumidamente os modelos de maturidade SOA analisados. A seção 3 discute a análise comparativa destes modelos. A seção 4 discute alguns trabalhos relacionados. Finalmente, a seção 5 apresenta algumas considerações finais e trabalhos futuros.

\section{Modelos de maturidade em SOA}

Modelos de maturidade [Drew 1992] em geral definem um conjunto de aspectos a serem analisados na avaliação do grau de amadurecimento de determinado objeto de estudo com relação a um contexto definido. 
No contexto corporativo, modelos de maturidade são ferramentas amplamente utilizadas na determinação do estado de maturidade atual de uma organização em certa área e para o planejamento das atividades necessárias para o alcance de um estágio de maturidade superior. No contexto de SOA, modelos de maturidade abordam níveis de maturidade distintos relacionados às definições e características de uma SOA.

$\mathrm{Na}$ indústria há uma grande variedade destes modelos. Cada modelo possui peculiaridades e visões corporativas distintas, porém, todos possuem o mesmo propósito geral: orientar as organizações na avaliação do nível de adequação a SOA, auxiliando no entendimento do estado atual e dos objetivos a serem alcançados para a adoção efetiva desta abordagem.

Os modelos de maturidade em SOA analisados são descritos brevemente a seguir. A escolha destes modelos é justificada pelo fato de serem propostos por grandes organizações que adquiriram experiência relevante ao longo dos anos concernente a implantação de SOA.

Modelo de Maturidade SOA da IBM. O modelo de Maturidade de Integração de Serviços (Service Integration Maturity Model- SIMM) [Brown et al. 2005] desenvolvido pela IBM em 2005, preconiza que a transição para uma SOA, seja através do reuso de processos já existentes ou pela definição de novos, pode ser realizada através de passos incrementais, sem que seja necessário analisar todos os processos e toda a infraestrutura de TI da organização simultaneamente.

Modelo de maturidade SOA da ORACLE. Proposto em 2006 [ORACLE 2006], é composto pelos domínios referentes à infraestrutura, arquitetura, informação e análise, operações, execução de projetos, finanças e portfólio, pessoas e organização e por fim, governança. A cada nível alcançado, alterações ocorrem nestes domínios de modo que sejam obtidos os resultados desejados na adoção de SOA.

Modelo de maturidade SOA da HP. O modelo tem o objetivo de avaliar o nível de maturidade atual de SOA da empresa mostrando como as diversas capacidades e ativos SOA podem amadurecer ao longo do tempo [Pugsley 2008]. De acordo com a experiência adquirida nas implantações de SOA realizadas pela HP, foram identificados oito modelos de domínio considerados os principais passíveis de adoção da abordagem em questão: negócios, gerenciamento do programa, governança, arquitetura, operação e gerenciamento, suprimento e demanda, pessoas e enfim permissão de tecnologias. Segundo Pugsley (2008), quando se é mestre em todos os domínios, há maior capacidade de efetivamente adotar e operar uma SOA, visto que as necessidades e as características destes serão levadas em consideração.

Modelo de maturidade SOA da SONIC. O New SOA Maturity Model (NSOAMM) [SONIC 2006] é específico para organizações que desejam implementar SOA baseada em Web Services. Este modelo caracteriza-se por apresentar os benefícios de negócio, as práticas e as metas chaves, os padrões relevantes selecionados e os fatores críticos de sucesso (tecnológicos e organizacionais) que compõem cada nível.

Modelo de maturidade SOA da BEA-IT. Denominado Modelo de Evolução SOA [BEA-IT 2006] é composto por três níveis de maturidade: Desenvolvimento de aplicações web, desenvolvimento de aplicações compostas e processos de negócios 
automatizados. No primeiro nível, a empresa que antes desenvolvia e integrava aplicações de forma tradicional, passa a oferecer soluções de negócio para setores internos e externos através de sites web para apoiar as necessidades de cada unidade de negócio. No segundo nível, desenvolvimento de aplicações compostas, um modelo de governança é estabelecido, as aplicações passam a se tornar mais flexíveis às mudanças e as informações são agregadas e fornecidas de forma composta. No terceiro nível, o gerenciamento de processos de negócios se torna o foco principal da empresa que busca a padronização destes e o alinhamento do negócio com TI.

Modelo de maturidade SOA do OPEN GROUP. O modelo de maturidade de integração de serviços do OPEN GROUP (OSIMM) [Open Group 2009] foi criado de forma colaborativa pela IBM, BEA-IT, Capgemini, HP, EDS, além de outros membros do OPEN GROUP, com o intuito de promover e apoiar a adoção de SOA e também como uma tentativa de padronizar os modelos de maturidade em SOA através da definição de um único modelo baseado em padrões abertos capaz de ser apoiado por tecnologias Open Source. O OSIMM, baseado no SIMM, define sete aspectos ou dimensões de uma organização que devem ser avaliadas para que o nível de maturidade seja definido. Tais dimensões são: negócios, organização e governança, métodos, aplicação, arquitetura, informação, infraestrutura e gerenciamento.

\section{Análise Comparativa dos Modelos de Maturidade SOA da Indústria}

Considerando a variedade de modelos existentes, é necessário conhecer as suas características, verificando as semelhanças e as distinções entre eles para que se consiga identificar quais são os pontos chaves considerados para a efetiva adoção de SOA. Para tanto, alguns parâmetros foram identificados para realizar a análise e apresentar as semelhanças e diferenças entre os modelos. Este artigo apresentará, por questão de espaço, apenas a análise de alguns dos parâmetros que foram considerados mais relevantes na identificação dos pontos chaves para adoção de SOA. Estes parâmetros são: objetivos da utilização de SOA, separação por níveis, estratégia de implantação de SOA, abrangência corporativa, tecnologias sugeridas, estratégia de alinhamento do negócio com TI e estratégia de reusabilidade de serviço. A análise completa pode ser consultada em [Oliveira 2011].

Alguns parâmetros iniciais foram identificados a partir de discussões com especialista da área de SOA e outros foram identificados ou refinados ao longo do processo de pesquisa.

É importante dizer também que os modelos analisados pertencem a empresas com fins comerciais, e que por isso pode causar um viés na pesquisa em termos do que é e não é importante na adoção de SOA.

Nas próximas subseções é apresentada a análise comparativa de acordo com os parâmetros identificados.

\subsection{Objetivos na Adoção de SOA}

O objetivo da utilização de SOA é muito importante para uma organização que deseja adotar esta abordagem. Observando os objetivos da utilização de SOA propostos pelas empresas fornecedoras de softwares que apóiam a adoção de uma Arquitetura Orientada 
a Serviços é possível analisar se tais objetivos estão em conformidade com aqueles a serem alcançados pela organização que pretende iniciar sua jornada em SOA.

Os objetivos da utilização de SOA propostos pela IBM são: desenvolver uma arquitetura bem definida e utilizável, reduzir custos operacionais e agilizar o negócio.

A ORACLE propõe como objetivo a redução de custos com integração, a ampliação de reutilização de ativos e o aumento da agilidade de negócios. O objetivo proposto pela HP é prover o melhor alinhamento entre negócios e TI e a execução de estratégias e metas de negócio a um custo mais aceitável.

A SONIC preconiza como objetivos o aumento da agilidade do negócio, a redução de custos e de riscos referentes à TI e o alcance da flexibilidade

Percebemos alguns objetivos em comum entre os modelos da IBM, ORACLE e SONIC, que visam a redução de custos e a agilidade de negócios. Porém, outros objetivos também são citados, como prover alinhamento entre negócios e TI e aumentar a flexibilidade das aplicações. Todas estas características são prometidas quando se pensa na adoção de SOA.

Analisar os objetivos dos modelos foi importante uma vez que permitiu melhorar os parâmetros de comparação da análise comparativa entre os modelos.

\subsection{Separação por Níveis}

O primeiro aspecto de semelhança comum a todos os modelos de maturidade SOA é a separação por níveis, que possibilitam mudanças gradativas ao longo do tempo de modo que riscos sejam minimizados através do planejamento estratégico de ações que provêem a adoção de SOA de maneira organizada. Deste modo, quanto mais elevado o nível de maturidade, maior a adequação da empresa aos conceitos de SOA.

A Tabela 1 mostra os níveis de cada modelo de maturidade analisado. Podemos perceber que o modelo da IBM é o que possui mais níveis (total de 7), enquanto o modelo da BEA-IT possui menos níveis (total de 3). Quanto maior o número de níveis, menor o esforço de pular de um nível para outro.

Um ponto observado com relação aos níveis dos modelos de maturidade pesquisados é a semelhança entre as características atribuídas ao primeiro nível de quase todos os modelos, no qual a integração é realizada sem um planejamento sistemático e os projetos implementados são simples, de baixo custo com foco apenas no conhecimento das tecnologias e na análise dos benefícios da adoção de SOA. Esta semelhança não se aplica ao primeiro nível do modelo de maturidade SOA do OPEN GROUP, que não aborda integração neste estágio, nem ao modelo de maturidade SOA da BEA-IT que aborda um grau de integração bastante elevado com relação aos demais modelos.

Outro ponto de semelhança comum a todos os modelos é o último nível de maturidade que em sua totalidade preconiza a automatização dos serviços que passam a responder automaticamente às requisições realizadas. Além disso, os serviços passam, teoricamente, a se combinar sem a intervenção humana para gerar novos serviços capazes de atender às solicitações.

Já foi afirmado anteriormente que a adoção de SOA exige um longo processo de adaptação tanto tecnológica quanto organizacional, logo, entende-se que SOA não pode 
ser alcançada de imediato. Josuttis (2007) reforça isto afirmando que SOA é uma estratégia que precisa ser introduzida na empresa gradualmente e que isto requer tempo uma vez que uma SOA totalmente desenvolvida exige muitas modificações. A preocupação em se adotar esta abordagem imediatamente pode acarretar a ausência de foco em requisitos essenciais para a implantação de SOA e na perda de alguns dos benefícios propostos por esta abordagem.

Os modelos de maturidade pesquisados em sua totalidade também sugerem como ponto chave a adoção gradativa de SOA através da definição de níveis que, ao logo do tempo e de acordo com as metas alcançadas, apresentam a visão de uma empresa mais amadurecida e adequada com relação aos conceitos de SOA.

Logo, pode-se concluir que o entendimento de adoção gradativa consiste em um ponto essencial para a obtenção de SOA.

Tabela 1. Níveis de maturidade dos modelos avaliados

\begin{tabular}{|c|c|c|c|c|c|c|}
\hline \multicolumn{7}{|c|}{ Modelo de maturidade SOA } \\
\hline Nível & IBM & ORACLE & HP & SONIC & OPEN GROUP & BEA-IT \\
\hline 1 & Silo & Oportunístico & Ad hoc & $\begin{array}{l}\text { Serviços } \\
\text { iniciais }\end{array}$ & Silo & $\begin{array}{c}\text { Desenvolvimento } \\
\text { de aplicações } \\
\text { web }\end{array}$ \\
\hline 2 & Integrado & Sistemático & Básico & $\begin{array}{c}\text { Serviços } \\
\text { arquitetados }\end{array}$ & Integrado & $\begin{array}{c}\text { Desenvolvimento } \\
\text { de aplicações } \\
\text { compostas }\end{array}$ \\
\hline 3. & Componentizado & Empresarial & Padronizado & $\begin{array}{c}\text { Serviços de } \\
\text { negócio/ } \\
\text { Serviços } \\
\text { colaborativos }\end{array}$ & Componentizado & $\begin{array}{l}\text { Processos de } \\
\text { negócios } \\
\text { automatizados }\end{array}$ \\
\hline 4. & $\begin{array}{c}\text { Serviços } \\
\text { simplificados }\end{array}$ & Mensurado & Gerenciado & $\begin{array}{l}\text { Serviços de } \\
\text { negócio } \\
\text { mensuráveis }\end{array}$ & Serviços & - \\
\hline 5. & $\begin{array}{l}\text { Serviços } \\
\text { compostos }\end{array}$ & Industrializado & Adaptado & $\begin{array}{l}\text { Serviços de } \\
\text { negócio } \\
\text { otimizados }\end{array}$ & $\begin{array}{l}\text { Serviços } \\
\text { compostos }\end{array}$ & - \\
\hline 6 & $\begin{array}{c}\text { Serviços } \\
\text { virtualizados }\end{array}$ & - & - & - & $\begin{array}{c}\text { Serviços } \\
\text { virtualizados }\end{array}$ & - \\
\hline 7 & $\begin{array}{c}\text { Serviços } \\
\text { reconfiguráveis } \\
\text { dinamicamente }\end{array}$ & - & - & - & $\begin{array}{c}\text { Serviços } \\
\text { reconfiguráveis } \\
\text { dinamicamente }\end{array}$ & - \\
\hline
\end{tabular}

\subsection{Abrangência Corporativa}

Com relação à abrangência corporativa, foi observado que tanto o modelo de maturidade SOA da IBM, quanto o da ORACLE, assim como também o modelo da HP e do OPEN GROUP abordam domínios, dimensões ou áreas de negócio que precisam ser avaliadas para a obtenção do nível atual de maturidade da organização. Para que um novo nível seja alcançado é necessário que algumas mudanças predeterminadas ocorram dentro destas áreas de modo que o grau de maturidade SOA da organização seja de fato visível. Quanto maior o grau de maturidade, mais elevado o nível de organização e de 
estruturação destas áreas. Embora cada modelo de maturidade trate as áreas por nomes distintos, como áreas de negócio, domínios do modelo e dimensões, o objetivo é semelhante: abordar domínios corporativos comumente existentes em grande parte das organizações.

Como diferenças gerais, são apresentados os seguintes aspectos: quantidade de domínios correspondentes a cada modelo, grau de enfoque dado à visão técnica, como por exemplo, arquitetura, infraestrutura, informação, permissão de tecnologias etc, e grau de enfoque atribuído à visão organizacional, tais como pessoas, negócios, governança, processos etc.

A Tabela 2 mostra as áreas que os modelos da IBM, ORACLE, HP e OPEN GROUP consideram. Os outros modelos não apresentaram estes conceitos. Podemos perceber que a única área em comum entre os modelos é a Arquitetura. Esta área tem como objetivo tratar as informações sobre o projeto arquitetural da SOA como um todo, dos serviços e das aplicações consumidoras que serão construídas [Dias 2010].

É importante notar que as áreas englobam aspectos técnicos e não técnicos, evidenciando que a adoção de SOA não é apenas a aquisição de tecnologias, e sim um processo que engloba várias dimensões da organização.

Tabela 2. Domínios/Dimensões/Áreas de negócio dos modelos de maturidade analisados

\begin{tabular}{|c|c|c|c|c|}
\hline \multirow{2}{*}{$\begin{array}{c}\text { Domínio/Dimensão/ } \\
\text { Área de Negócio }\end{array}$} & \multicolumn{4}{|c|}{ Modelo de Maturidade SOA } \\
\hline & IBM & ORACLE & HP & OPEN GROUP \\
\hline Negócios & $\mathbf{x}$ & & $\mathrm{x}$ & $\mathrm{x}$ \\
\hline Arquitetura & $\mathrm{x}$ & $\mathrm{x}$ & $\mathrm{x}$ & $\mathrm{x}$ \\
\hline Organização & $\mathbf{x}$ & $\mathbf{x}$ & & $\mathrm{x}$ \\
\hline Métodos & $\mathrm{x}$ & & & $\mathrm{x}$ \\
\hline Processos & $\mathrm{x}$ & & & \\
\hline Governança & & $\mathbf{x}$ & $\mathrm{x}$ & $\mathrm{x}$ \\
\hline Aplicações & $\mathrm{x}$ & & & $\mathrm{x}$ \\
\hline Infraestrutura & $\mathrm{x}$ & $\mathrm{x}$ & & $\mathbf{x}$ \\
\hline Operações & & $\mathrm{x}$ & $\mathrm{x}$ & \\
\hline Informação & $\mathrm{x}$ & $\mathrm{x}$ & & $\mathbf{x}$ \\
\hline Finanças e portfólio & & $\mathrm{x}$ & & \\
\hline Execução de projetos & & $\mathrm{x}$ & & \\
\hline Pessoas & & $\mathrm{x}$ & $\mathrm{x}$ & \\
\hline Suprimento e demanda & & & $\mathrm{x}$ & \\
\hline Permissão de tecnologias & & & $\mathrm{x}$ & \\
\hline
\end{tabular}

\subsection{Estratégia de Alinhamento entre TI e o Negócio}

O alinhamento do negócio com a tecnologia da informação diz respeito ao atendimento eficiente dos objetivos de negócio da organização através do gerenciamento também 
eficiente das suas aplicações. Tal adequação deve envolver a integração entre a estratégia de negócio e estratégia de TI, assim como a infraestrutura de negócio e a infraestrutura de TI [Chan 2002].

Existem diversas estratégias que possibilitam a busca do alinhamento entre o negócio e TI, dentre estas pode ser citado o Gerenciamento de Processos de Negócio (BPM - Business Process Management) [Moore 2003] e Governança SOA [Afshar 2007].

A governança é responsável por garantir que as políticas estabelecidas com relação às aplicações sejam cumpridas em conformidade com os conceitos de SOA no processo de desenvolvimento e de gerenciamento dos serviços.

Os modelos de maturidade da IBM e da HP sugerem a utilização de governança SOA e o BPM como estratégia de alinhamento entre negócio e TI.

O modelo de maturidade da ORACLE sugere como estratégia de alinhamento o investimento em governança, a utilização de ferramentas para integração e orquestração de serviços (BPEL), o uso de ferramentas de modelagem e de padronização de processos de negócio (BPMN), a ênfase em métricas para mensurar a variação de processos e a utilização de ferramentas de gerenciamento para monitorar processos, políticas e eventos.

Os modelos de maturidade da SONIC, BEA-IT e OPEN GROUP sugerem o gerenciamento de processos de negócio, a especificação de políticas para a criação e modificação destes processos, a utilização de governança SOA para o alinhamento entre negócio e TI e o Monitoramento de Atividades de Negócio (BAM) para armazenamento e análise de informações estatísticas referente aos processos de negócio.

Analisando-se os modelos de maturidade SOA citados, percebe-se que todos se assemelham por preconizarem o alinhamento entre TI e o negócio e por sugerirem o investimento em governança SOA e BPM como estratégia no seu alcance.

Governança SOA é definida como o planejamento de todo o processo de implantação e de continuidade de uma SOA em uma organização. Ela é a responsável por fornecer diretrizes necessárias e por garantir que decisões a serem tomadas com relação a todos os termos técnicos e não técnicos que englobam esta abordagem não remeterão a crises econômicas ou à perda de controle dos objetivos definidos, dos recursos utilizados ou dos serviços desenvolvidos.

Josuttis (2007) e Manes (2007) ressaltam que a governança possui quatro fundamentos principais: políticas, processos, métricas e organização. As políticas definem que as ações devem ser realizadas de forma correta, os processos reforçam as políticas, as métricas provêem uma visão concernente ao cumprimento das políticas e o reforço necessário para que estas sejam seguidas e a organização é responsável por estabelecer uma cultura que suporte o processo de governança, e.g. [Josutis 2007], [Manes 2007].

$\mathrm{Na}$ adoção de SOA, os fundamentos citados são aplicados de forma contínua para o alcance dos benefícios propostos por esta abordagem. Neste contexto, são focados tanto aspectos organizacionais (como finanças, definição de papéis e de responsabilidades, processos, padrões, etc.) quanto aspectos técnicos (como tecnologias, documentação, gerenciamento e monitoramento de serviços, entre outros). Portanto, 
pode-se afirmar que investimentos em governança consistem em um ponto chave para que SOA seja obtida com sucesso.

BPM é uma abordagem disciplinada de gerenciamento suportada por tecnologias com foco na melhoria de processos, tratando-os como ativos que podem ser gerenciados e adaptados às constantes mudanças [Gartner 2009].

A adoção de BPM permite a identificação, a modelagem, o controle e a melhoria de processos de negócio com base na análise real de como estes são normalmente executados ("as-is") e das ações necessárias para que estes sejam modificados e aplicados ("to-be") para o alcance dos objetivos definidos.

No contexto de SOA, o gerenciamento de processos apresenta-se como uma atividade de grande importância, visto que serviços podem representar atividades, subprocessos ou processos de negócio, logo, é necessário a utilização de uma abordagem sistemática para identificá-los, modelá-los, implementá-los, reusá-los e para apoiar às mudanças as quais esses estão sujeitos.

\subsection{Estratégia de implantação de SOA}

A definição de estratégias durante a obtenção de um novo paradigma arquitetural consiste em uma atividade essencial para a obtenção de sucesso. As pessoas são peças fundamentais para que tais mudanças aconteçam, para que políticas sejam aceitas e praticadas e por fim para que haja a efetivação dos planos traçados na adoção desta nova abordagem. Portanto, a adoção de SOA exige o envolvimento de desenvolvedores, analistas de negócios e arquitetos capacitados, além do incentivo da alta gerência e da educação da organização no todo, já que há uma grande mudança nas estruturas corporativa e tecnológica durante este processo.

A necessidade deste requisito para a obtenção de SOA foi observada com base na análise dos modelos de maturidade pesquisados que em conjunto sugerem governança como principal estratégia de implantação de SOA através da definição de políticas que permitam a sua adoção e continuidade.

O modelo de maturidade da ORACLE além de sugerir o investimento em governança sugere também a criação de um centro de excelência em SOA (que consiste em uma equipe responsável por entender, compartilhar e implantar os conceitos relacionados à Arquitetura Orientada a Serviços), recompensas e incentivos em troca da adesão à SOA, alocação de custos para o uso de recursos de TI e expansão de financiamento para o centro de excelência SOA e apoio executivo das iniciativas SOA.

A HP, semelhantemente a IBM, sugere o investimento em governança, além de treinamento em SOA para equipes de TI e para todos os funcionários. A SONIC sugere Governança, patrocínio da alta gerência e a formação de um centro de competência SOA liderado por um grupo de arquitetos. O OPEN GROUP propõe governança, treinamento relacionado às mudanças arquiteturais e a contratação de recursos especializados. A BEA-IT propõe governança, investimento em uma equipe com boas habilidades técnicas e apoio da alta gerência.

\subsection{Estratégia de reusabilidade de serviços}

A reusabilidade de serviço refere-se à utilização dos mesmos serviços em aplicações distintas sem a necessidade de sua reimplementação, desse modo, segundo Josuttis 
(2007) quando os sistemas precisam utilizar uma funcionalidade, eles podem acessá-la requisitando o mesmo serviço. Isto evita que o desenvolvimento se torne uma atividade redundante, diminuindo os custos e o tempo inerentes ao desenvolvimento, e aumentando a qualidade das aplicações.

Diante destes benefícios e considerando que a reusabilidade é uma das principais características de SOA, verifica-se a necessidade de observar quais modelos de maturidade abordam uma estratégia concernente a este requisito.

Baseado na pesquisa é possível perceber que a única estratégia identificada em alguns dos modelos analisados, mais especificamente o modelo do OPEN GROUP, da ORACLE e da HP, é a utilização de um registro de serviços para que estes sejam localizados e reutilizados. Não foi identificada na documentação dos modelos analisados nenhuma outra estratégia explícita para reutilização de serviços. Entretanto, é importante se pensar nesta questão uma vez que é através da efetiva reutilização de serviços que se conseguirá a redução de custos no desenvolvimento de novas aplicações.

\subsection{Tecnologias Sugeridas}

SOA não é uma tecnologia, e sim uma abordagem que necessita de tecnologias para apoiá-la. A importância de saber quais tecnologias são sugeridas pela empresa proponente do modelo de maturidade se dá pela necessidade da empresa iniciadora em SOA verificar a possibilidade de adoção das tecnologias propostas, considerando a viabilidade de aprendizado destas, a aquisição de pessoas qualificadas para implementar as aplicações e o impacto da implantação destas na organização. Além disso, é necessário verificar se a mudança de paradigma tecnológico agrega de fato valor ao negócio. Tal relevância é ainda maior quando a empresa possui sistemas legados, ou seja, sistemas de grande importância compostos por tecnologias obsoletas.

Considerando que o nível de maturidade SOA da organização também será avaliado de acordo com o amadurecimento da tecnologia adotada, pode ser relevante implantar um modelo de maturidade SOA com uma perspectiva tecnológica semelhante aquela a ser adotada pela empresa iniciadora em SOA, no entanto isto não é uma regra.

A ORACLE, HP, SONIC, OPEN GROUP e BE-IT não especificam nenhuma linguagem, porém sugere a utilização de Web Services como tecnologia principal para a adoção de SOA, diferentemente da IBM que especifica a utilização de JEE (Java Enterprise Edition) ou .NET.

\section{Trabalhos Relacionados}

Rathfelder e Groenda (2008) relatam alguns modelos de maturidade SOA propostos por grandes indústrias e propõem um novo modelo denominado Modelo de Maturidade Independente (iSOAMM) que, segundo os autores, pode ser aplicado sem a dependência de tecnologias e de produtos.

Meier (2006) em sua tese realiza uma análise sobre o modelo de maturidade da SONIC e o modelo de maturidade da ORACLE e a partir da combinação dos benefícios destes propõe um novo modelo denominado Modelo de Maturidade SOA Combinado (CSOAMM) composto por dez níveis de maturidade com o objetivo principal de auxiliar as empresas a compreender os pontos necessários para a adoção de SOA. 
O diferencial entre o trabalho proposto e os citados anteriormente é que este não tenciona a análise de modelos de maturidade SOA com vista na proposta de um novo, antes tem por principal objetivo analisar cada modelo pesquisado e a partir desta análise identificar os pontos chaves considerados como principais por estes modelos para a adoção de SOA.

A quantidade e os tipos de requisitos identificados no processo de análise, assim como o número de modelos comparados (seis), também consistem em um diferencial relevante por fornecerem subsídios consistentes para o atendimento do objetivo proposto.

\section{Considerações Finais}

Este artigo destinou-se a apresentar uma análise de modelos de maturidade em SOA propostos na indústria e a identificar, baseado nesta análise, os principais pontos chaves para a adoção de SOA. Os modelos analisados foram: Modelo de maturidade SOA da IBM, modelo de maturidade SOA da ORACLE, modelo de maturidade SOA da HP, modelo de maturidade SOA da SONIC, modelo de maturidade SOA da BEA-IT e modelo de maturidade do OPEN GROUP.

Este artigo permitiu identificar quais são os pontos chaves que as empresas devem considerar quando pensar em adotar SOA: Adoção gradativa de SOA; consideração de diferentes dimensões como arquitetura, pessoas, negócios, organização governança, infraestrutura etc na evolução da adoção; Governança SOA como estratégia para garantir as políticas estabelecidas; BPM como estratégia de alinhamento entre negócio e TI; Envolvimento e educação de pessoas, como peças fundamentais para que as mudanças organizacionais aconteçam; investimento de recursos e criação de um centro de excelência SOA para apoiar a iniciativa de adoção; utilização de ferramentas, por exemplo, um registro para catalogação dos serviços para que possam ser reutilizados.

Como trabalho futuro é proposto o estudo de relatos de empresas que utilizaram algum dos modelos de maturidade pesquisados e a análise da influência destes na prática para o sucesso na adoção de SOA. Além disso, é importante realizar uma revisão sistemática da literatura para identificar evidências da relevância destes pontos chaves na adoção.

\section{Referências}

Afshar, M. "SOA Governance: Framework and Best Practices, Version 1.1", Oracle, May 2007.

BEA-IT, SOA Evolution Model (2006). SOA Blueprint. Disponível em: http://soablueprint.com/yahoo_site_admin/assets/docs/SOAEvolutionModel.2911007 53.pdf. Referência Principal.

Brown W. A. Johnston K. S.; Larsen G.; Palistrant, J.(2005). "SOA Development Using the IBM Rational Software. Development Platform: A Practical Guide”. IBM Corporation. 
CHAN, Yolande E. (2002). "Why haven't we mastered alignment? The importance of the informal organization structure". MIS Quarterly Executive.

Dias, J. (2010). A Software Architecture Process for SOA Definition - Designing Service-Oriented Architectures in an Enterprise Context. LAP Lambert Academic Publishing.

Drew, D.W. "Tailoring the software engineering Institute's (SEI) Capability Maturity Model (CMM) to a software sustaining engineering organization". Proceedings of Conference on Software Maintenance, 1992.

Gartner, Group. (2009). "Business Process Management Program Key Initiative Overview".

Josuttis, N. (2007) SOA in Practice - The Art of Distributed System Design. O'Reilly Media.

Meier, F. (2006). "Service Oriented Architecture Maturity Models - A guide to SOA adoption". Master's thesis, University of Sk"ovde, School of Humanities and Informatics.

Moore, J., "Business process management", Chemical Engineering Process, American, 2003, pp. 22-23.

Nasr, K., Gross, H., Deursen, A. (2010). Adopting and Evaluating Service Oriented Architecture in Industry. 14th European Conference on Software Maintenance and Reengineering.

Niemann, M; Eckert, J; Repp, N; Steinmetz, R.(2008). “Towards a generic governance model for service-oriented architectures". In: Proceedings of the Fourteenth Americas Conference on Information Systems, Toronto.

OASIS (2006). "Reference Model For Service Oriented Architecture”. Disponível em < http://www.oasis-open.org/committees/download.php/16587/wd-soa-rm-cd1ED.pdf>

Oliveira, J. Modelos de Maturidade em SOA: Uma análise comparativa da indústria. Monografia (graduação) - Universidade Federal da Paraíba/CCAE, Rio Tinto, 2011.

ORACLE. (2006) SOA Maturity Cheat Sheet. Disponível em: <http://pt.scribd.com/doc/2890015/oraclesoamaturitymodelcheatsheet>

Pugsley, Andrew. (2008). "Assessing your programming SOA. HP Worldwide SOA Service Program". Disponível em: <http://h20195.www2.hp.com/v2/GetPDF.aspx/4AA0-4824ENW.pdf $>$.

Rathfelder, C; Groenda, H.(2008). “iSOAMM: An Independent SOA Maturity Model”. In Proc. of 8th IFIP International Conference on Distributed Applications and Interoperable Systems (DAIS'08), volume 5053/2008 of Lecture Notes in Computer Science, pages 1-15. Springer-Verlag Berlin Heidelberg.

SONIC (2006), Movin SOA on up: Introducing a New Service-Oriented Architecture Maturity Model. Disponível em: http://web.progress.com/en/movin-soa-on-up.html.

The Open Group (2009). Service Integration Maturity Model (OSIMM). 2009. Disponível

em: https://www.opengroup.org/projects/osimm/uploads/40/17990/OSIMM_v0.3a.pdf 